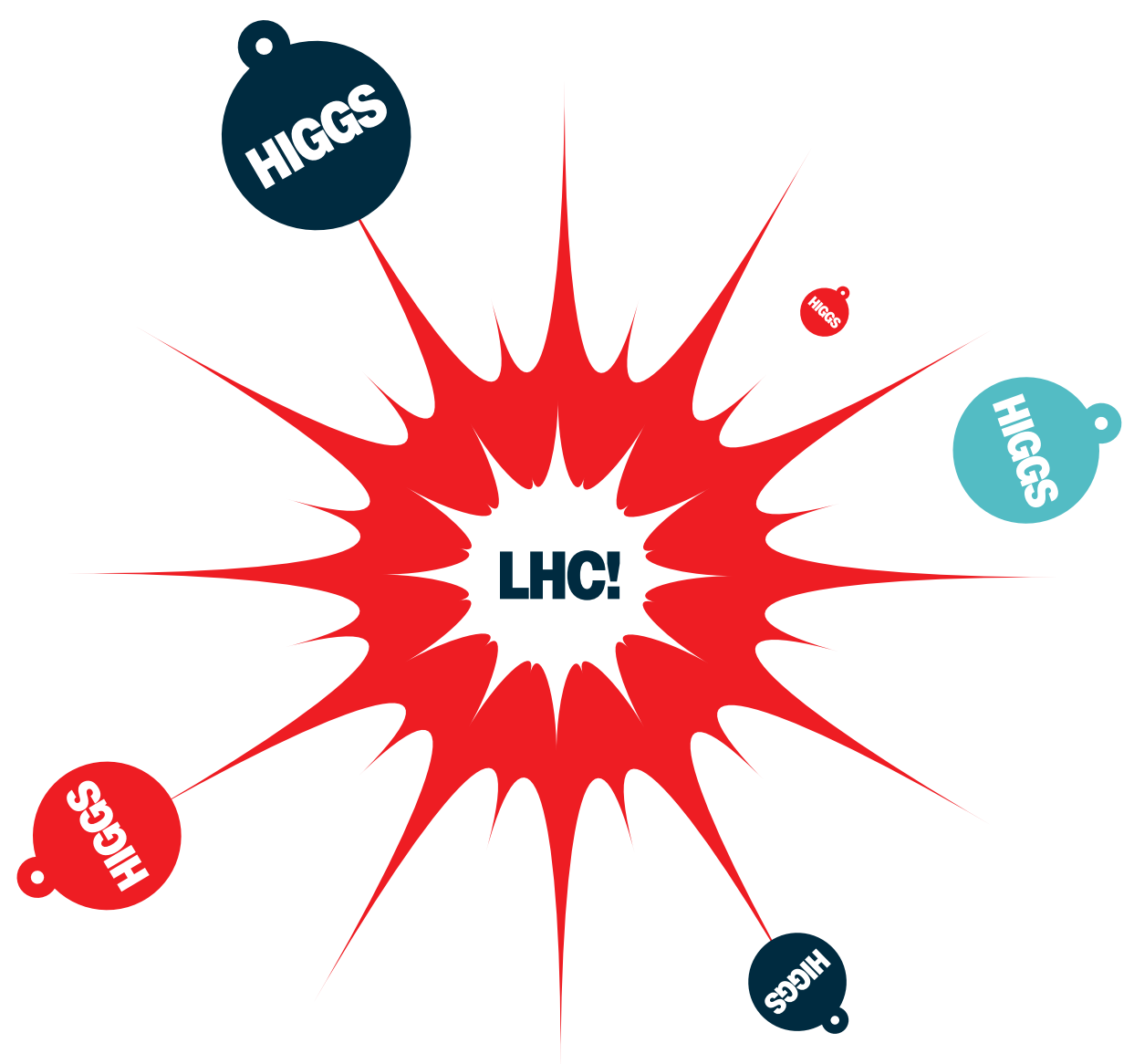

\title{
re \\ Unification +150
}

\section{In 1861, James Clerk Maxwell unified electricity, magnetism and light. Experiments under way today could inch physicists closer to combining everything else.}

BY M. MITCHELL WALDROP

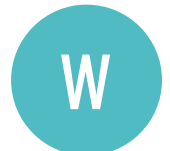

hen it happens - if it happens - don't look for Hollywoodstyle drama. Physicists at the Large Hadron Collider (LHC) outside Geneva in Switzerland won't suddenly gasp with astonishment, and their monitors won't flash the message, "Higgs boson detected."

Instead, the discovery will unfold over the course of months. Computers will trawl through petabytes $\left(10^{15}\right.$ bytes) of collision data in search of a handful of distinctive events that might signal their quarry's existence, while physicists cross-check every candidate. Only when they have accumulated enough events to be sure - maybe a dozen - will they publicly proclaim the discovery of the sought-after Higgs.

Even so, the announcement will be dramatic - and timely. Exactly 150 years ago, the
Scottish physicist James Clerk Maxwell showed that three apparently separate phenomena electricity, magnetism and light — are different aspects of one phenomenon, today known as electromagnetism (see page 289). The Higgs discovery could take that unification a giant step further by filling in the last and most critical piece of the 'standard model', an extension of Maxwell's equations that encompasses three of the four forces of nature: electromagnetism and the weak and strong forces that act on subatomic particles. The Higgs boson is thought to interact with electrons, quarks and other fundamental particles, endowing them with mass - and thus making it possible for the standard model to describe the Universe as we know it.

This puts the standard model as it is today in the same position as Maxwell's theory before experiments demonstrated the existence of electromagnetic waves, says Frank Wilczek, 
a physicist at the Massachusetts Institute of Technology in Cambridge, and co-recipient of the 2004 Nobel Prize in Physics for his part in creating the model. "It looks good, lots of its predictions have been verified, but the most dramatic new thing remains to be verified."

But even if the Higgs boson is discovered as predicted, physicists will not be satisfied. The ultimate goal is a unification theory that would reveal how all observed particles and forces are just different manifestations of a single underlying system, which can be expressed within a common mathematical framework. Such an elegant result is not possible with the standard model, which includes the strong force that binds the atomic nucleus only as an afterthought, and has nothing at all to say about gravity. The standard model also has no explanation for dark matter, an invisible substance that outweighs the ordinary matter in stars and galaxies by a factor of roughly five.

Although physicists agree that some kind of larger unification is needed, they don't know what form that should take. For four decades, nearly as long as the standard model has existed, researchers have been speculating about ways to extend it with exotic ideas such as supersymmetry, extra dimensions and holographic space-time. "The situation is that there are a bunch of hypotheses on the table, most of them not new, with no experimental support for any of them," says Lee Smolin, a physicist at the Perimeter Institute for Theoretical Physics in Waterloo, Canada.

"The good news," says Smolin, "is that the experiments are finally being done." Within a few years, thanks to the LHC and other experiments, physicists should have a much clearer idea of which theoretical notions are real and will take their place in the ultimate unification.

\section{SUPERSYMMETRY}

If the Higgs boson turns out to be exactly what is predicted from the standard model, it will have zero internal angular momentum ('spin-0'), and a mass somewhere between 115 and 180 billion electron volts $(\mathrm{GeV})$ in the energy units favoured by particle physicists. But such a match would be pretty boring, says John Ellis, a theoretical physicist at King's College London. It would be much more fun, he thinks, if the LHC physicists didn't find anything. "After all those years of speculating, we finally look under the appropriate lamp post, and it's not there at all!" says Ellis. That would force the theorists back to the drawing board, "but there are various drawing boards to go back to". There could be more complicated ways of generating mass, or something more unexpected. "That would be very exciting," says Ellis.

Alternatively, he says, it is entirely possible that the LHC will turn up not one Higgs particle, but a whole family of them. That would be a sign of supersymmetry, a theory that predicts a zoo of as-yet unobserved 'superparticles', one to match each of the 25 particles in the standard model - the force-carrying bosons such as photons, gluons and the Higgs, and the fermions, such as quarks and electrons, which make up matter. These superpartners would be heavy - at least $600 \mathrm{GeV}$.

Supersymmetry appeals to physicists because it provides a unified mathematical description of bosons and fermions, which otherwise seem utterly unrelated. And the theory would greatly strengthen the case for a 'grand unification' of the strong, weak and electromagnetic forces, leaving only gravity unexplained.

In the standard model, the strong force's interaction strength - expressed in terms of a constant analogous to electric charge - is very different from the strengths of the weak and electromagnetic forces. But if supersymmetry is assumed to be true, quantum corrections show all three strengths to be exactly equal - just as would be expected if the forces are actually one.

Supersymmetry would also solve some problems of other grand unified theories, such

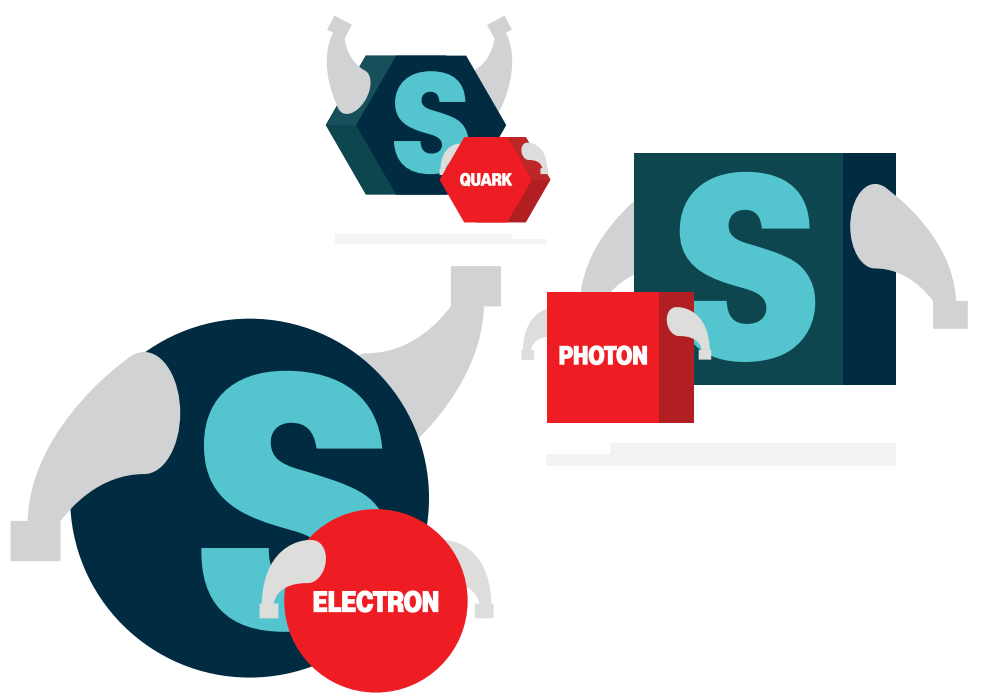

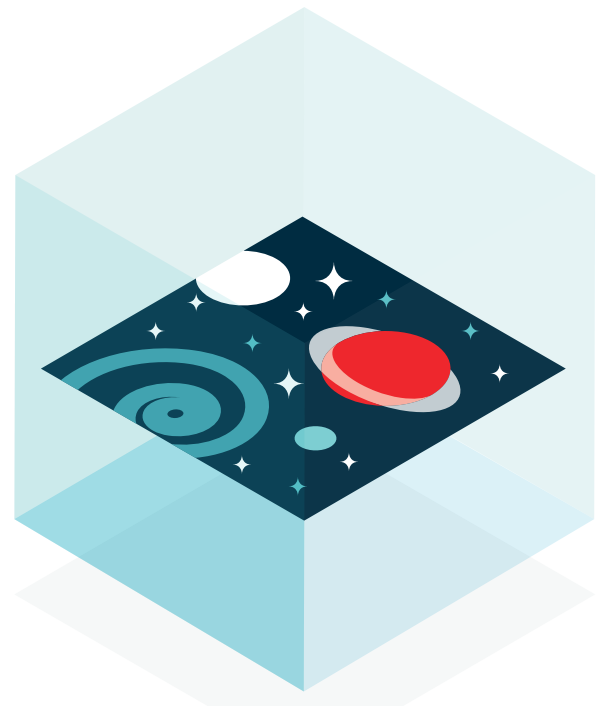

as their predictions that the proton should be unstable. The presence of the superpartners in calculations tends to suppress proton decay, leading to a decay rate far below the limits currently set by experiment.

Finally, and perhaps most importantly from an observational standpoint, supersymmetry might very well provide an explanation for dark matter. This invisible cosmic haze behaves like a swarm of massive particles that interact very weakly with ordinary atoms, and has so far been detected only by its gravitational influence on visible stars and galaxies. No particle in the standard model has the right properties - but several of their predicted superpartners do. If one of them is indeed the dark-matter particle, it may soon be observed not only at the LHC, but also in one or more of the dark-matter detectors now operating around the world (see Nature doi:10.1038/news.2011.125;2011).

But just because supersymmetry promises many wonderful solutions to current problems, that's no guarantee it is true. "If supersymmetry is seen, that's absolutely fabulous," says Smolin. "But if not - well, that will be fabulous, too. It's always better to know."

\section{EXTRA DIMENSIONS}

Conversations with LHC physicists can become surreal - especially when they start talking with a straight face about finding that staple of science fiction, extra dimensions.

One big reason that they take this prospect seriously is that extra dimensions are predicted by string theory, by far the most popular attempt at unification beyond the standard model. String theory posits that the fundamental particles are actually vibrating threads of energy. Since it was developed in the late 1960s, string theory has remained a mental exercise, with no physical evidence to back it up. But it has proved remarkably compelling even so. It predicts the existence of forces that look a lot like the strong, weak and electromagnetic forces of the standard 
model. It incorporates supersymmetry in a natural way. And it automatically includes gravity: string-theory equations show that closed loops of string would behave like gravitons, the particles postulated to carry the force of gravity. "It combines all the principles of physics we know," says Nathan Seiberg, a string theorist at the Institute for Advanced Study in Princeton, New Jersey. "That's huge. There's no other suggestion that even comes close." The extra dimensions arise because string theory has its most natural formulation in 11 dimensions, only 4 of which would be observable to us: the 3 dimensions of space and 1 of time. The missing dimensions are easy to explain, says Seiberg: the theory allows them to be so tightly rolled up that they're invisible under ordinary circumstances.

The LHC could detect those extra dimensions if the particles generated by collisions have enough energy - and therefore shortenough quantum wavelengths - to start spiralling around those tightly curled dimensions. The energy of that spiralling would show up as mass, according to Einstein's famous relativity theorem. So LHC physicists could detect whole families of higher-mass duplicates of the standard-model particles.

An alternative scenario, also an outgrowth of string theory, suggests that what we perceive as three-dimensional space is actually a kind of membrane floating in a higher-dimensional space. We never notice the extra directions because all the particles of the standard model are confined to the membrane. But LHC collisions might be energetic enough to let in a burst of gravitational energy from outside our membrane. The result would be a jet of collision products spraying off to one side of the collision point, but apparently with nothing to balance it on the other side - as if the jet had been hit by a bolt out of nowhere.

LHC physicists have calculated the experimental signatures of all these extra-dimensional phenomena and more, says Albert de Roeck, deputy spokesman of the Compact Muon Solenoid experiment, one of the LHC's two big collision detectors. "But the moving target is to know the scale" of the extra dimensions, he says. If they are rolled up too tightly, on a scale smaller than $10^{-19}$ metres, then the energy required to probe them will be beyond the LHC's reach.

That is a distinct possibility, which is why physicists consider it a long shot to observe extra dimensions at the LHC. "I'm not holding my breath," says Michael Duff, a physicist at Imperial College London. "My bet is that if there are extra dimensions, they're at the Planck scale" of $10^{-35}$ metres, where quantum mechanics and gravity are thought to unite in some still-unknown way.

Nonetheless, the pursuit of extra dimensions is considered well worth the effort.
Their existence would not prove string theory correct; it is perfectly possible to have extra dimensions without strings. But confirming one of its major predictions would considerably bolster the case for string theory.

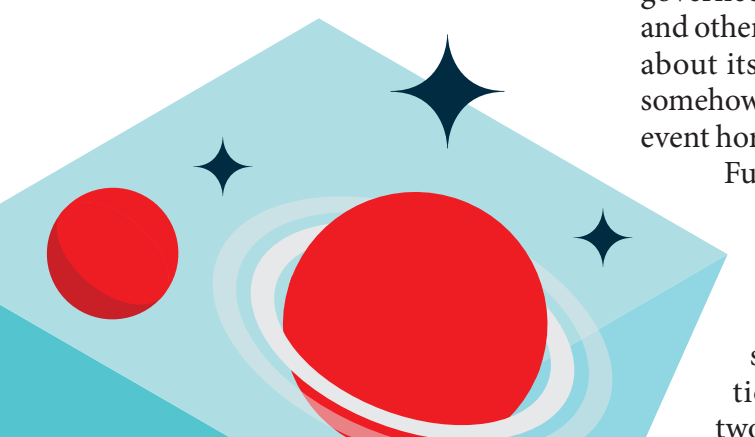

there is no way for outside observers to get any information from a black hole, because anything closer to the black hole than a surface known as the event horizon is cut off from the rest of the Universe. So if a black hole is governed by thermodynamics, as Hawking and others demonstrated, all the information about its three-dimensional interior must somehow be encoded on its two-dimensional vent horizon.

Furthermore, after decades of analysis and generalization of this argument, many physicists now believe that it applies to any three-dimensional volume, from black holes to empty space: the volume's entire information content can be encoded in its two-dimensional surface. Or to put it another way, the ultimate unified theory of everything should describe our apparently solid three-dimensional world in terms of a lower-dimensional reality. Our Universe would emerge from the theory like a three-dimensional optical image from a twodimensional hologram.

Although this 'holographic principle' might be an element of some ultimate unified theory, it does not by itself say what that theory should be. And not every physicist buys it. "It's interesting and provocative, but extremely vague," says Wilczek. Mathematically, at least, a version of the holographic principle does apply in a string-theory model known as AdS/CFT duality, in which it has been studied extensively. And perhaps more importantly, it might be possible to test the idea.

Craig Hogan, director of the Fermilab Center for Particle Astrophysics in Batavia, Illinois, has suggested that if the holographic principle is true, quantum effects could produce a kind of 'holographic noise' in light beams. The effect would be minute, says Hogan, but might be detectable by the kind of ultraprecise laser interferometers already used in gravitational wave experiments.

He and his colleagues are already building hardware to test whether this effect will work as expected, says Hogan, and if it does, they hope to proceed next year with a full-scale experiment, projected to cost about US $\$ 2$ million over three years. "That's cheap, as these things go," says Hogan - and that is probably just as well. "These are tests of physics we don't know, so by definition, it's exploratory".

Among all the theories that could help to unify physics - from strings to holography and even more esoteric concepts - there is the possibility that many may turn out to be the same idea, viewed from different perspectives. But the only way to find out is to do the tests.

"The LHC gives us hope for a huge leap," says Seiberg. "In a few years we should be a lot smarter." — SEE EDITORIALP.265

M. Mitchell Waldrop is an editor for Nature in Washington DC. 